\title{
The giant aortic root aneurysm related to bicuspid aortic valve treated with valve-sparing operation
}

\author{
Radosław Gocoł ${ }^{1}$, Aleksandra Żak ${ }^{1}$, Joanna Ciosek ${ }^{2}$, Marek A. Deja ${ }^{1,3}$ \\ ${ }^{1}$ Department of Cardiac Surgery, Upper-Silesian Heart Center, Katowice, Poland \\ ${ }^{2} 3^{\text {rd }}$ Division of Cardiology and Structural Heart Diseases, \\ Upper-Silesian Heart Center, Katowice, Poland \\ ${ }^{3}$ Department of Cardiac Surgery, Medical University of Silesia, \\ School of Medicine, Katowice, Poland
}

A 30-year-old patient was admitted with dyspnea and severe chest pain radiating to the left scapula. Transesophageal echocardiography (TEE) showed bicuspid aortic valve (BAV) with severe eccentric regurgitation jet along anterior mitral leaflet (Fig. 1A), aneurysm of the root and ascending aorta with maximum diameter of $130 \mathrm{~mm}$ without dissection (Fig. 1B). The aortic ring was $34 \mathrm{~mm}$, left ventricular ejection fraction (EF) 50\% and end-diastolic volume (EDV) $280 \mathrm{~mL}$. Angiocomputed tomography showed an enormous aneurysm $(130 \times 110 \mathrm{~mm}$, Fig. $1 \mathrm{C})$ involving aortic root and the ascending aorta with normal $34 \mathrm{~mm}$ aortic arch. Urgent aortic valve sparing operation modo David was performed. TTE on post-op
7 showed no aortic regurgitation, aortic root of $36 \mathrm{~mm}$ (Fig. 1D, E, F), EF 30\% and EDV $206 \mathrm{~mL}$. Subsequently the patient was discharged home. Six months later TTE showed no aortic regurgitation, improvement of $\mathrm{EF}$ to $60 \%$, and $\mathrm{EDV}$ reduction to $180 \mathrm{~mL}$. Despite the large dilatation of the aortic root and aortic ring, the valve leaflets retained their original structure, which allowed for aortic valve sparing. The key element of the operation was to select the right size of the aortic prosthesis, in order to restore physiological relationship between the aortic orifice and aortic cusps. This case demonstrated that the size of aortic root alone cannot constitute a contraindication to the valve sparing procedure.

Conflict of interest: None declared

Address for correspondence: Radosław Gocoł, MD, Department of Cardiac Surgery, Upper Silesian Heart Center, ul. Ziołowa 45/47, 40-635 Katowice, Poland, tel: +48 605401558, e-mail: gocot@poczta.onet.pl 


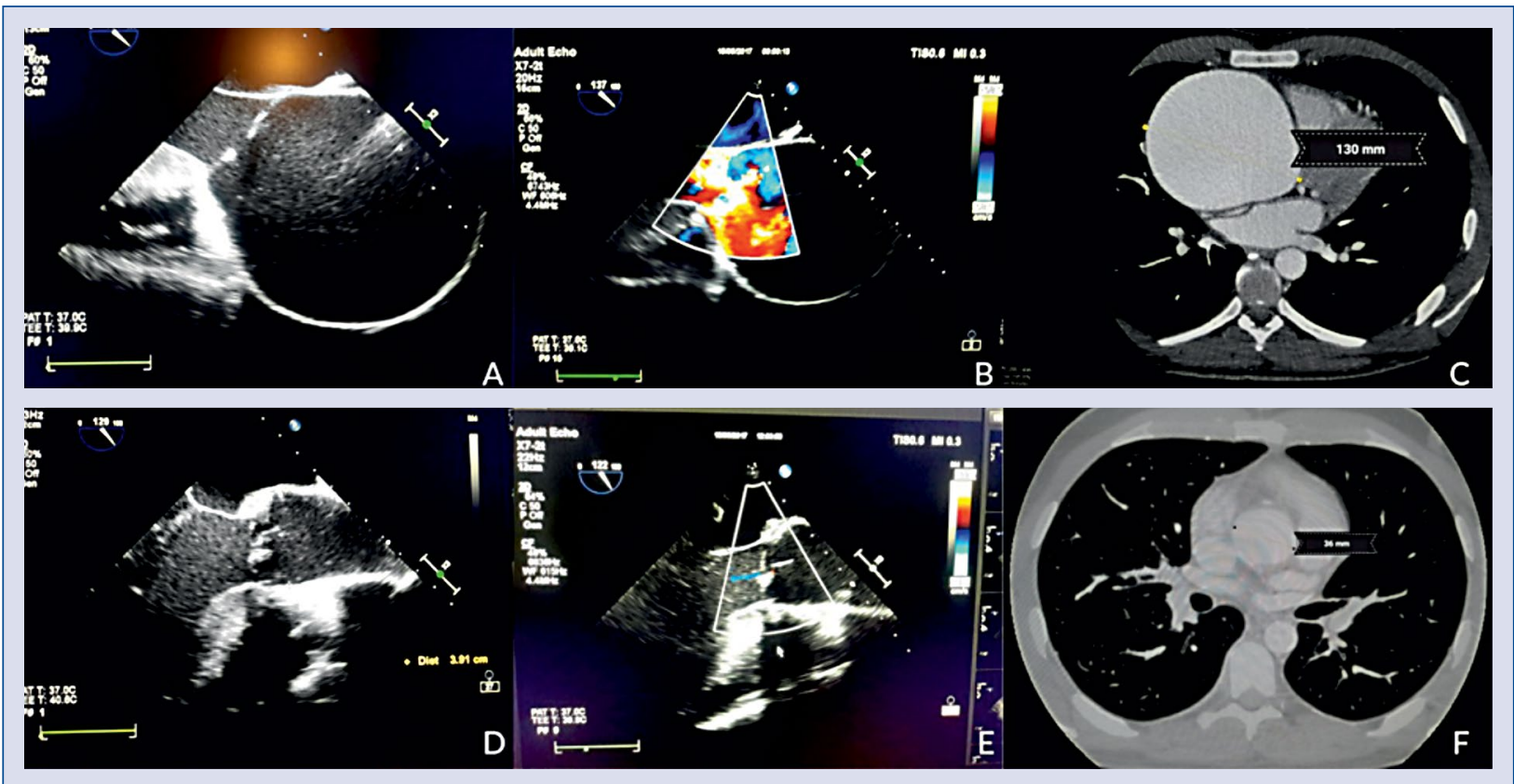

Figure 1. Preoperative transesophageal echocardiography (TEE) showing $130 \mathrm{~mm}$ aortic root and ascending aorta aneurysm (A) and severe aortic regurgitation (B); C. Preoperative angio-computed tomography (angio-CT): $130 \mathrm{~mm}$ aortic root and ascending aorta aneurysm. Postoperative TEE showing the root and ascending aorta (D) and trace aortic regurgitation (E); F. Postoperative angio-CT showing the aortic root. 